\title{
COVID-19 pandemic-the learning continues
}

\author{
Pranav Bansal ${ }^{1}$, Kirti Ahuja², Saru Singh ${ }^{3}$, Shivani Sharma ${ }^{4}$ \\ 1-Professor \& Head; 2-Assistant Professor, 3-Associate Professor \\ Department of Anaesthesiology \& Critical Care, BPS Govt Medical College, Khanpur Kalan, Sonipat, Haryana, India. \\ 4-Professor, Dept. of Physiology, Medical University of the Americas, St. Kitts \& Nevis.
}

\section{Abstract}

\begin{abstract}
The Covid-19 pandemic is the most severe global health crisis of our time and greatest challenge to mankind after World War II. A combination of therapies including vitamins, hydroxychloroquine, steroids, plasma therapy and immunomodulators have proved to be effective in symptomatic cases but no specific drug or vaccine has been developed to fight covid-19 till date. Till the time new vaccine rolls out, trials are evaluating the efficacy of BCG in fighting against Covid-19. The Covid-19 with its high propensity of transmission, especially through asymptomatic carriers has been spreading rapidly since the outbreak of the disease. With the existing lacunas in ongoing preventive and management strategies and increasing risk of community transmission, the role of development of herd immunity in control of covid-19 can play a major role.
\end{abstract}

Key words: Covid-19, Corona virus, Tocilizumab, Hydroxychloroquine, Plasma therapy

Citation: Bansal P, Ahuja K, Singh S, Sharma S. Anaesth. pain \& intensive care 2020;24(3):259-262.

Received: 4 Jul 2020; Reviewed: 5 Jul 2020; Accepted; 7 Jul 20

Since Dec 2019, the new strain of Corona virus knocked the doors of this planet and the whole medical fraternity and heath regulatory authorities have been working $24 \times 7$ to eradicate this pandemic. Presently, more than $17,500,000$ positive cases have been reported in approximately 213 countries worldwide, with more than 10,827,000 cases being recovered and more than 6.7 lac deaths reported worldwide officially. ${ }^{1}$ The unofficial count of covid-19 infection and deaths is still an enigma due to massive underreporting of cases in many developing countries. The case fatality rate of Covid-19 is estimated to be vary between $0.1-4.5 \%$, in different countries with an exceptionally high mortality rate of $14 \%$ in Italy and no exact explanation for these variations. ${ }^{2}$ Moreover, it is yet not ascertained whether the corona virus infection was the cause of deaths in these cases or it was an incidental infection in a terminally ill or comorbid patient.

In the past few months, medical journals have been flooded with uncountable articles on covid-19, only to provide subtle benefit as the definitive treatments are yet unknown and vaccines still under trial phase.
Currently, the management guidelines for covid-19 are being revised very frequently by various health regulatory bodies in accordance with the findings of small-scaled studies and trials. Frequent organization of webinars are not helping much to the already overinformed medical practitioners, thus adding to utter chaos! For ignorant and poor patients, magical potions offered by local quacks are providing wonderful results and magical cure rates, while on the contrary many multi-specialty and corporate hospitals are facing charges of medical negligence following death of patients. Thousands of health care workers have already succumbed to death worldwide, while performing their duties in Covid wards and ICU's and many more might perish by the time this pandemic waves-off.

Despite, no specific proven treatment, patients are being administered various cock-tails of off-label drugs with varying results. Based on limited available evidence, current clinical approaches consider the combination of antiviral and immune-active drugs. The protective role of Vitamins B, C and D is yet not established though the same are being prescribed 
freely by many medical personals in management of mild cases. By the month of March 2020, the sales of an anti-malarial drug, hydroxychloroquine (HCQ) had sky-rocketed owing to its antiviral and immunemodulating activity. The drug received emergency use authorization from FDA at the end of March, for prophylaxis against covid-19. On June 15, FDA revoked this authorization, citing studies indicating that HCQ didn't significantly decreased mortality and may have caused serious health risks by increasing the QT interval. ${ }^{3}$ Recently, WHO also has discontinued the treatment arms of HCQ and lopinavir/ ritonavir combination in the management of hospitalized patients after the data of Solidarity Trial revealed the same results. ${ }^{4}$

On May 1, the antiviral drug Remdesivir (GS 5734), received emergency use authorization in patients with moderate symptoms of covid-19, after its encouraging results in WHO's ongoing Solidarity Trial. $^{5}$ Remdesivir may help in reducing the viral load but they definitely are not the "game changers".

Favipiravir triphosphate, a purine nucleoside analogue, acts as a competitive inhibitor of RNA-dependent RNA polymerase is being increasingly used in covid19 management and has shown to reduce the virus clearance times when compared to HCQ or lopinavir/ ritonavir combination. ${ }^{6}$

Tocilizumab (Actemra ${ }^{\mathrm{TM}}$ ), a monoclonal antibody that acts by binding to IL- 6 receptor has been reported to be effective against covid-19 induced cytokine storm. It is being used off-label in patients with moderate symptoms and progressively increasing oxygen requirements or mechanically ventilated patients not showing improvement despite use of steroids. The drug has shown to have significant reduction in mortality and favourable outcomes if given early in covid pneumonia with ARDS. ${ }^{7}$ Similarly, type I interferon, IFN-beta, is also currently being evaluated in REMAP-CAP and the WHO's Solidarity Trial to tackle severe COVID-19. ${ }^{8}$

Recently, the RECOVERY Trial in UK has revealed that low-cost dexamethasone decreased the risk of death by $35 \%$ (one third) in patients on ventilation and $20 \%$ (one fifth) in patients on oxygen, leading to a $17 \%$ reduction in the total 28 -day mortality rate, but not among patients not receiving respiratory support. ${ }^{9}$ The UK government has recently authorized the state- funded National Health Service (NHS) to use the steroid dexamethasone in severe cases.

Besides, the benefits of using convalescent plasma therapy are yet to be ascertained in RECOVERY trial, India has authorized its off-label use in patients with moderate disease that are not improving (oxygen requirement is progressively increasing) despite use of steroids. ${ }^{10}$ Convalescent plasma therapy seeks to make use of the antibodies developed in the recovered patient against the coronavirus. The therapy is meant for critical patients with low oxygen saturation levels, or those suffering a cytokine storm and extreme breathlessness with severe pneumonia.

Since there is ongoing controversy regarding the pathophysiology behind Covid pneumonia, the condition is preceded by a stage called 'happy hypoxia' or 'silent hypoxia' where patients do not complain of any respiratory difficulty despite "alarmingly low" oxygen levels and oxygen saturations running as low as 50 percent. ${ }^{10}$ The patients reach in a critical condition in hospitals and require starting of early oxygen therapy along with low molecular weight heparin (enoxaparin) to reverse the widespread microvascular thrombosis, observed in this disease. ${ }^{11}$

Unpredictable mortalities witnessed with Covid-19 infection initially favored the conventional approach of early intubation and ventilation, but quickly faded away due to unfavorable outcomes and high mortalities. Initial guidelines issued from various pulmonology and critical care societies discouraged the use of non-invasive ventilation (NIV) due to high risk of aerosol generation and cross-contamination to health care workers. ${ }^{12}$ But the recent guidelines encourage the use of NIV and high-frequency nasal oxygenation (HFNO) due to improved outcomes in many critical patients. The recent inclusion of helmet mask (also known as Bubble helmet) in clinical practice for delivering high flow oxygen has been claimed to reduce the propagation of aerosols in the patient care surroundings. ${ }^{13,14}$

A new scoring system to assess the severity of chest inflammatory changes on CT-scan can has been developed by the radiologists in the Netherlands. This CO-RADS classification system predicts pulmonary involvement in patients with moderate to severe symptoms in categories $1-5$, with category 1 denoting 
very low chance of infection and category 5 indicating typical features of covid-19 involvement. ${ }^{15}$

The use of prone positioning for oxygenation and ventilation in awake or intubated patients has also shown short-term improvement in ventilationperfusion matching by decreasing gravity-related atelectasis and aiding in lung recruitment. This has led to a rise in oxygen saturations by $4-10 \%$. Prone positioning has been advised for atleast 12 hours in patients on ventilator support, though the mean duration of positioning in awake patients has been nearly 8 hours.

Other therapies include lose dose radiation therapy (LDRT) for treatment of covid pneumonia of are under trials. Single dose of radiation (30-100cGy) to the lung can incite anti-inflammatory properties by decreasing the levels of cytokines, and decreasing the symptoms of viral pneumonia, but its efficacy in decreasing overall mortality is yet to be discovered. ${ }^{16}$

To completely stop the spread of this epidemic, there is an urgent need of a potent vaccine or either herdimmunity. There are some 21 vaccine projects around the world in various stages of development and clinical trials. On $12^{\text {th }}$ July, the Sechenov First Moscow State University of Russia successfully completed the human trials of $1^{\text {st }}$ covid vaccine. The Gamalei Institute of Epidemiology and Microbiology of the university has confirmed the safety of this vaccine. However, it seems unreasonable to predict that any of these vaccines will hit the commercial markets before January 2021.

Till date, the re-infection rate with Covid-19 is estimated to be near zero, and one-time infection is presumed to provide short term immunity for at least 6 months. Currently, trials evaluating the efficacy of BCG vaccine in preventing Covid-19 infection are under way in Australia, Spain, France and Netherlands. WHO has not recommended BCG vaccination as it has non-specific effects on immune system and its efficacy in fighting Corona virus is unknown?

The researchers and medical faculties across the world are working full throttle in attempts to save more and more lives. Despite, the vast availability of researches, advanced diagnostic modalities and pharmacotherapies, we were living in an illusion of being well equipped to fight any disease. Indeed, any new strain of a mutated virus can create a world-wide menace by challenging our immune systems and medical advancements, thus making us realize that we will never be adequately prepared to fight the nature.

Today, even a common man is aware of terms like Corona virus, N95 mask and PPE kit as they are witnessing the one of its kind lock-down and quarantine of their lifetimes. Currently, a more preventive approach in social gatherings and public places is still the best measure till affordable specific treatments are made available to general public at costs. Studies need to be perfomed for evaluating seropositivity in local population against Covid-19, so that areas are identified where the role of herd immunity has come into play. Many questions still remain unanswered to date, like the probability of infection in health care workers, the infectious dose, possibility of re-infection, long-term immunity following infection, etc. but the learning still continues.

\section{Conflict of interest}

Nil declared by the authors.

\section{Authors' contribution}

All authors contribute equally in the concept, search of literature and preparation of the manuscript.

\section{References}

1. Worldometer.info/Coronavirus [Internet]. 2020 [updated 2020 July 13; cited 2020 July 13]. Available from: https://www.worldometers.info/coronavirus

2. Global change data lab. Case fatality rate of the ongoing COVID-19 pandemic [Internet]. Oxford: Global change data lab; 2020 [updated 2020 July 12; cited 2020 July 13]. Available from: https://ourworldindata.org/grapher/coronavirus-cfr

3. Food and Drug Administration. Coronavirus (COVID-19) Update: FDA revokes emergency use authorization for chloroquine and hydroxychloroquine [Internet]. Washington: Food and Drug Administration; 2020 [updated 2020 June 15; cited 2020 July 13]. Available from: https://www.fda.gov/news-events/pressannouncements/coronavirus-covid-19-update-fdarevokes-emergency-use-authorization-chloroquine-and hydroxychloquinine 
4. World Health Organization. WHO discontinues hydroxychloroquine and lopinavir/ritonavir treatment arms for COVID-19. Geneva: World Health Organization. 2020. Available from: https://www.who.int/newsroom/detail/04-07-2020-who-discontinueshydroxychloroquine-and-lopinavir-ritonavir-treatmentarms-for-covid-19

5. Food and Drug Administration. Coronavirus (COVID-19) Update: FDA issues emergency use authorization for potential COVID-19 treatment [Internet]. Washington: Food and Drug Administration; 2020 [updated 2020 May 1; cited 2020 July 13]. Available from: https://www.fda.gov/news-events/pressannouncements/coronavirus-covid-19-update-fdaissues-emergency-use-authorization-potential-covid-19 treatment

6. De A. Explained: The drugs being used for treating Covid19 symptoms in India [Internet] New Delhi: The Indian Express;2020 [Updated 2020 June 30; cited 2020 July 13]. Available from: https://indianexpress.com/article/explained/coronaviruscovid-19-medicines-treatment-available-in-indiaremdesivir-favipiravir-hcq-6474185/

7. Capra R, Rossi ND, Mattioli F, Romanelli G, Scarpazza C, Sormani MP, et al. Impact of low dose tocilizumab on mortality rate in patients with COVID-19 related pneumonia. Eur J Intern Med. 2020; 76:31-35. [PubMed] [Free Full Text] DOI: 10.1016/j.ejim.2020.05.009

8. Jalkanen J, Hollmen M, Jalkanen S. Interferon beta-1a for COVID-19: critical importance of the administration route. Crit Care. 2020;24: 335. [PubMed] [Free Full Text] DOI: 10.1186/s13054-020-03048-5

9. World Health Organization. WHO welcomes preliminary results about dexamethasone use in treating critically ill COVID-19 patients [Internet]. Geneva: World Health Organization; 2020 [Updated 2020 June 16;cited 2020 July 13]. Available from: https://www.who.int/news- room/detail/16-06-2020-who-welcomes-preliminaryresults-about-dexamethasone-use-in-treating-criticallyill-covid-19-patients

10. Das KL. Convalescent Plasma Therapy: A treatment for COVID-19 [Internet]. Noida: India legal live; 2020 [updated 2020 June 27; cited 2020 July 13]. Available from: $\quad$ https://www.indialegallive.com/specialstory/convalescent-plasma-therapy-a-treatment-forcovid-19

11. Tobin MJ, Laghi F, Jubran A. Why COVID-19 silent hypoxemia is baffling to physicians? Am J Respir Crit Care Med. 2020;10:1164. [Free Full Text] DOI: 10.1164/rccm.202006-2157CP

12. Atallah B, Mallah SI, AlMahmeed W. Anticoagulation in COVID-19. Eur Heart J Cardiovasc Pharmacother. 2020;10:1093. [PubMed] [Free Full Text] DOI: 10.1093/ehjcvp/pvaa036

13. Carter C, Aedy H, Notter J. COVID-19 disease: NonInvasive Ventilation and high frequency nasal oxygenation. Clinics in Integrated Care. 2020;1:100006. [Free Full Text] DOI:10.1016/j.intcar.2020.100006

14. Lyons $\mathrm{C}$, Callaghan M. Apnoeic oxygenation in paediatric anaesthesia: a narrative review. Anaesthesia. 2020;10:1111. DOI:10.1111/anae.15107

15. Prokop M, Everdingen WV, Vellinga TVR, Ufford JQV, Stoger L, Beenen L, et al. CO-RADS -A categorical CT assessment scheme for patients with suspected COVID19: definition and evaluation. Radiology.2020;201473 [PubMed] [Free Full Text] DOI: 10.1148/radiol.2020201473

16. REMOVED

17. Kirkby $C$, Mackenzie $M$. Is low dose radiation therapy a potential treatment for COVID-19 pneumonia? Radiother Oncol. 2020;147:221. [PubMed] [Free Full Text] DOI: 10.1016/j.radonc.2020.04.004 